\title{
Editorial \\ Axillary Surgery in DCIS: Is Less More?
}

\author{
Monica Morrow, MD \\ Breast Surgery Service, Memorial Sloan-Kettering Cancer Center, 1275 York Avenue, New York, NY 10065, USA
}

As our understanding of the biology of ductal carcinoma in situ (DCIS) has increased, the management approach to the axilla has evolved. DCIS, by definition, cannot metastasize, and it has been known for many years that nodal metastases, presumed to be due to unrecognized invasion, are extremely uncommon. In a National Cancer Database review of 10,946 patients with DCIS undergoing axillary dissection between 1985 and 1991, only 3.6\% were found to have axillary metastases, ${ }^{1}$ and this number is even lower when only cases of screen-detected DCIS are considered. The major impetus for nodal staging in DCIS today is the recognized sampling error that occurs when needle biopsy techniques are used for diagnosis. In 1998, a joint committee of the American College of Surgeons, American College of Radiologists, and College of American Pathologists developed a standard for the diagnosis and management of DCIS which concluded that axillary dissection was unnecessary for the majority of patients with DCIS. Consideration of a level I dissection to avoid a second surgical procedure for patients undergoing mastectomy for extensive or high-grade DCIS was recommended. ${ }^{2}$ When these guidelines were updated in $1992,{ }^{3}$ they reflected both the emergence of sentinel node biopsy as an axillary staging technique and the low likelihood of identifying invasive carcinoma with nodal disease in localized DCIS by recommending that axillary staging in patients treated with breastconserving approaches be reserved for those found to have invasion on final pathologic evaluation. In patients undergoing mastectomy, sentinel node biopsy for axillary staging was recommended for

Published online August 5, 2008.

Address correspondence and reprint requests to: Monica Morrow, MD; E-mail: morrowm@mskcc.org

Published by Springer Science+Business Media, LLC $\odot 2008$ The Society of Surgical Oncology, Inc. surgeons experienced in the technique; for those who were not, a level I axillary dissection was suggested.

In this issue of the Annals of Surgical Oncology, Porembka et al. ${ }^{4}$ use Surveillance, Epidemiology, and End Results (SEER) data from 1988 through 2002 to analyze axillary management in patients with DCIS. Based on their analysis of 23,502 patients, they conclude that there is persistent and excessive utilization of axillary nodal assessment, particularly axillary dissection, in patients with DCIS. The data as presented is alarming, with $21 \%$ of those having surgery between 1998 and 2002 undergoing lymph node assessment. In the group that had breast-conserving surgery (BCS), $67 \%$ of the nodal procedures were axillary dissections; in the mastectomy group, $87 \%$ had axillary dissection. There are several important caveats to these observations. First, the SEER database, while an excellent source of high-quality, population-based cancer data, may not be an ideal source for analyzing the nuances of axillary surgery. SEER coding rules state that, if axillary nodes are present in a mastectomy specimen, the procedure should be coded as a modified radical mastectomy rather than a simple mastectomy. The removal of a few level 1 axillary nodes in order to ensure removal of the axillary tail of the breast is not uncommon, and certainly does not constitute a therapeutic axillary dissection, although it would be coded as such by SEER rules. Thus, the most accurate assessment of the use of axillary dissection is obtained from the subset of women undergoing BCS. Based on the authors' statement that $70 \%$ of the 10,637 women treated in the 1998-2002 time period had BCS, and that 589 had axillary staging, this results in about 395 axillary dissections, or $5 \%$ of the total group.

The second major issue regarding this work is that the time period under study was the same time period in which the sentinel node biopsy technique was being validated, refined, and disseminated. Two major 
clinical trials of sentinel node biopsy began patient accrual in the USA in 1999, and sentinel node biopsyspecific procedure codes only became available in 1999. Since that time, the body of evidence supporting the use of sentinel node biopsy as a safe and effective technique for axillary staging has expanded exponentially, and rates of sentinel node biopsy use in 2002 have limited relevance to practice patterns in 2008. Chen et al. ${ }^{5}$ used data from 490,899 patients with stage I and T2N0 stage 2 invasive carcinoma in the National Cancer Database to examine trends in the use of sentinel node biopsy and found that patients diagnosed in 2005 had an odds ratio of 5.26 [95\% confidence interval (CI) 5.13-5.40] for sentinel node biopsy compared with those diagnosed in 1998. In this study, $65.5 \%$ of axillary staging in 2005 was performed by sentinel node biopsy.

However, in spite of the increased use of sentinel node biopsy today compared with in 2002, the overarching question that Porembka et al. bring to our attention, the question of the appropriateness of any axillary staging in the patient with DCIS, is one worthy of serious consideration. There is clearly no indication for axillary dissection as a staging procedure in the patient with DCIS. Ample opportunities exist for practising surgeons to learn the sentinel node biopsy technique, and it is a routine part of residency training; not knowing how to perform the procedure is no longer a relevant excuse. It is equally apparent that routine sentinel node biopsy in all patients with DCIS is also not indicated. Data from the National Surgical Adjuvant Breast and Bowel Project (NSABP) B17 and B24 trials document that the risk of axillary recurrence in patients treated with breast-conserving surgery, with or without radiation therapy and with or without tamoxifen, is extremely low. In the NSABP B17 trial the axillary recurrence rate was 0.83 per 1000 patient years, and in NSABP B24 the rate was 0.36 per 1000 patient years. ${ }^{6}$ These low rates of axillary recurrence make it impossible to justify the routine use of a procedure that is associated with a 7\% incidence of lymphedema and a $9 \%$ incidence of paresthesia 6 months postoperatively, as demonstrated in the American College of Surgeons Z10 trial. $^{7}$ A similar incidence of arm sequelae at 1-year follow-up has been reported in the Axillary Lymphatic Mapping against Nodal Axillary Clearance (ALMANAC) trial. ${ }^{8}$ It is well recognized that the likelihood of identifying invasive carcinoma is higher in DCIS which is associated with a clinical or mammographic mass, in highgrade lesions, and in lesions of larger size; however, the majority of patients with DCIS with these features do not have coexisting invasive carcinoma. At present, a validated model to identify subsets of patients at truly high risk for invasive cancer does not exist. In light of this, in patients treated with breast-conserving surgery, limiting sentinel node biopsy to those who have invasive carcinoma in the lumpectomy specimen is cost effective and minimizes unnecessary morbidity. The situation in patients undergoing mastectomy is somewhat different. The risk of sampling error is highest in patients with large areas of DCIS, and the opportunity for sentinel node biopsy is lost once mastectomy is performed, changing the risk-benefit ratio to favor sentinel node biopsy, as suggested in current guidelines. $^{9}$

The initial enthusiasm for the use of sentinel node biopsy in the patient with DCIS anytime there is an axilla in the vicinity should now be tempered by the availability of high-quality empiric data from clinical trials documenting both excellent outcomes in patients with DCIS who do not undergo axillary staging, and the small but real incidence of long-term sequelae in patients who undergo the procedure. When tempted to use sentinel node biopsy in a majority of patients with DCIS "just to be safe," it is worth remembering that a fool with a tool is still a fool.

\section{REFERENCES}

1. Winchester DP, Menck HR, Osteen RT, et al. Treatment trends for ductal carcinoma in situ of the breast. Ann Surg Oncol 1995; 2:207-13.

2. Winchester DP, Strom EA. Standards for diagnosis and management of ductal carcinoma in situ (DCIS) of the breast. American College of Radiology. American College of Surgeons. College of American Pathologists. Society of Surgical Oncology. CA Cancer J Clin 1998; 48:108-28.

3. Morrow M, Strom EA, Bassett LW, et al. Standard for the management of ductal carcinoma in situ of the breast (DCIS). CA Cancer J Clin 2002; 52:256-76.

4. Porembka MR, Abraham RL, Sefko JA, et al. Factors Associated with lymph node assessment in ductal carcinoma in situ: Analysis of 1998-2002 SEER data. Ann Surg Oncol 2008 (This issue).

5. Chen AY, Halpern MT, Schrag NM, et al. Disparities and trends in sentinel lymph node biopsy among early-stage breast cancer patients (1998-2005). J Natl Cancer Inst 2008; 100:462-74.

6. Julian TB, Land SR, Fourchotte V, et al. Is sentinel node biopsy necessary in conservatively treated DCIS? Ann Surg Oncol 2007; 14:2202-8.

7. Wilke LG, McCall LM, Posther KE, et al. Surgical complications associated with sentinel lymph node biopsy: results from a prospective international cooperative group trial. Ann Surg Oncol 2006; 13:491-500.

8. Mansel RE, Fallowfield L, Kissin M, et al. Randomized multicenter trial of sentinel node biopsy versus standard axillary treatment in operable breast cancer: the ALMANAC Trial. J Natl Cancer Inst 2006; 98:599-609.

9. Morrow M, Harris JR. Practice guideline for breast conservation therapy in the management of invasive breast cancer. $J \mathrm{Am}$ Coll Surg 2007; 205:362-76. 